\title{
Magnetic fluctuations and itinerant ferromagnetism in two-dimensional systems with van Hove singularities
}

\author{
P. A. Igoshev ${ }^{a)}$ A. A. Katanin ${ }^{\mathrm{a}), \mathrm{b})}$, and V. Yu. Irkhin ${ }^{\mathrm{a})}$
}

November 30, 2018

a) Institute of Metal Physics 620041, Ekaterinburg, Russia

b) Max-Planck-Institut für Festkörperforschung, 70569 Stuttgart, Germany

\begin{abstract}
We use the quasistatic approach to analyze the criterion of ferromagnetism for two-dimensional (2D) systems with the Fermi level near Van Hove singularities (VHS) of the electronic spectrum. It is shown that the spectrum of spin excitations (paramagnons) is positively defined when the interaction $I$ between electrons and paramagnons, which corresponds to the Hubbard on-site repulsion $U$, is sufficiently large. The critical interactions $I_{c}$ and $U_{c}$ remain finite at Van Hove filling and exceed considerably their values obtained from the Stoner criterion due to incommensurate spin fluctuations, which are important near the ferromagnetic quantum phase transition. Combining the quasistatic approximation and the equation of motion method for the Green function we obtain the results for the electronic self-energy to first order in the inverse number of spin components.
\end{abstract}




\section{Introduction}

Two-dimensional and layered systems with strong electronic correlations have been attracting attention of researchers for more than two decades. In such systems, in particular, the $\mathrm{CuO}_{2}$ planes of the $\mathrm{La}_{2-x} \mathrm{Sr}_{x} \mathrm{CuO}_{4}$ and $\mathrm{YBa}_{2} \mathrm{Cu}_{3} \mathrm{O}_{7-\delta}$ compounds the low dimensionality favors appearance of the high- $T_{c}$ singlet superconductivity [1]. On the other hand, a number of low-dimensional triplet type superconducting compounds $\left(\mathrm{Sr}_{2} \mathrm{RuO}_{4}, \mathrm{UGe}_{2}\right)$ have been discovered recently. For example, the compound $\mathrm{UGe}_{2}$ is ferromagnetic at low temperatures and pressure. It is expected that the ferromagnetic fluctuations may play a crucial role in the properties of the paramagnetic compound $\mathrm{Sr}_{2} \mathrm{RuO}_{4}$ [2]. This is confirmed by the properties of the La-doped compound $\mathrm{La}_{x} \mathrm{Sr}_{2-x} \mathrm{RuO}_{4}$ which is near the ferromagnetic transition at $x=0.27$ [3], and also by the properties of the isoelectronic compound $\mathrm{Ca}_{2} \mathrm{RuO}_{4}$ [4], which becomes ferromagnetic under pressure. The above-mentioned compounds have Van Hove singularity (VHS) of the density of states near the Fermi level, the description of magnetic fluctuations in the presence of these singularities is an important problem, which may serve as a basis for deeper insight into magnetic and superconducting properties of these compounds.

According to the Stoner theory, the large value of the density of states at the Fermi level, occurring due to VHS, leads to a possibility of ferromagnetically ordered ground state. The Stoner theory, however, neglects fluctuations of magnetic order parameter, which may significantly change the necessary conditions for ferromagnetism. It is well known that for systems with nonsingular density of states the Stoner theory does not explain correctly magnetic and thermodynamic properties, e.g. it predicts larger temperatures of magnetic phase transition in comparison with the experimental data. Starting from the papers by Murata, Doniach [5] and Dzyaloshinskii [6], the paramagnon theory has been developed to account for the effect of spin fluctuations and describe the properties of weak- and nearly ferromagnetic materials. Later on this theory was systematically formulated by Moriya [7]. This theory considers magnetic excitations (paramagnons), which give the dominant contribution to thermodynamic properties of weak ferro- and antiferromagnets. Properties of paramagnons in the vicinity of quantum phase transitions were investigated later within the renormalization group method by Hertz in the framework of the $\phi^{4}$-model [8], the corresponding results were generalized by Millis to finite temperatures [9]. The applicability of the Hertz-Moriya-Millis theory in two- and three dimensions has been, however, recently questioned, because the lowest order corrections to the spin susceptibility were shown to be non-analytical with respect to momentum and therefore dramatically change the spectrum of magnetic excitations [10].

In the presence of VHS in the vicinity of the Fermi level the Hertz-Moriya-Millis theory itself, however, is not applicable to describe the ferromagnetic ground state. Indeed, due to the influence of VHS the noninteracting magnetic susceptibility $\chi_{q}^{0}$ may not have a maximum at the point $q=0$ [11]. Since the spectrum of paramagnons in the Hertz-MoriyaMillis theory is determined by the inverse spin susceptibility $\left(\chi_{q}^{0}\right)^{-1}$, this spectrum is not positively defined in the presence of VHS and the ground state with the spin density wave at the wavevector $\mathbf{Q} \neq 0$ becomes more preferrable in this theory. Due to the presence of the electron-electron interaction, however, the paramagnon subsystem is not independent, but it is interacting with the electronic degrres of freedom, which can change the position of the spin susceptibility maximum in momentum space. Since the Hertz-Moriya-Millis theory neglects the renormalization of the momentum dependence of the susceptibility by the electron-paramagnon interaction, it cannot therefore describe correctly ground state properties in the presence of VHS. Furthermore, for the position of the Fermi level in the 
vicinity of the VHS the paramagnon interaction energy in the Hertz-Moriya-Millis theory is negative, which corresponds to attraction between paramagnons with the possibility of bound states formation. The magnitude of this interaction may be also changed essentially by higher-order terms in the electron-paramagnon interaction $I$, which can result in the repulsive paramagnon interaction.

Therefore, to investigate the possibility of the ferromagnetic ordering in the presence of VHS it is necessary to take into account the effect of the electron-paramagnon interaction on the spin susceptibility more accurately than in the existing spin-fluctuation theories. An attempt to go beyond these theories was made by Hertz and Klenin [12], who considered the sum of infinite series of diagrams for irreducible susceptibility. However, the renormalization of the momentum dependence of the susceptibility, which becomes crucial in the presence of VHS, was not considered in their study. The aim of the present paper is to formulate a consistent spin-fluctuation theory in the presence of VHS and to investigate their influence on the possibility of ferromagnetism.

To this end, we consider the spin-fermion model, which treats interacting electronic and spin degrees of freedom. This model was introduced in early 1990's to treat antiferromagnetic fluctuations in high- $T_{c}$ superconductors [13] (see also Refs. [14, 15]), but can be generalized to the case of ferromagnetic fluctuations [16]. Since previous attempts to derive this model from the Hubbard model met the problem of the so-called Fritz ambiguity, we use alternative way to derive this model and obtain the relation between the parameters of the Hubbard and spin-fermion models. Having this relation, the phase diagram of these models can be investigated. It is argued that for large enough electron-paramagnon interaction $I_{c}$ (and the corresponding Hubbard on-site Coulomb repulsion $U_{c}$ ) the spectrum of paramagnons becomes positively defined, which corresponds to the possibility of ferromagnetism at $I>I_{c}$. The quantities $I_{c}\left(U_{c}\right)$ exceed substantially the corresponding values, obtained from the Stoner criterion. The latter is in an agreement with the results of the functional group approach [17] and the numerical investigations for the Hubbard model. The critical values of the interaction $I_{c}\left(U_{c}\right)$ are finite at the Van Hove filling.

The plan of the paper is the following. In Section 2 we introduce the spinfermion model and derive the "generalized" Stoner criterion for ferromagnetism. In Sections 3.1 and 3.2 the application of this criterion for investigation of the phase diagram and magnetic properties of the model is presented. The additional criterion of ferromagnetism, requiring the positivity of the energy of the paramagnon interaction is considered in Section 3.3 and showed to be less important than the generalized Stoner criterion. In Section 3.4 we combine the quasistatic approach with the equation of motion method for the electronic Green function which allows us to investigate electronic properties of the system. The obtained results are discussed in Section 4. In Appendix A we discuss the derivation of the spin-fermion model from the Hubbard model. The diagram technique for the spin-fermion model is presented in Appendix B. The formulae for the second derivative of magnetic susceptibility with respect to the momenta are presented in Appendix C. 


\section{Magnetic Properties of Two-Dimensional Systems}

\subsection{The Hubbard model and an applicability of the Stoner crite- rion of ferromagnetism}

To investigate the effect of VHS on magnetic properties of $2 \mathrm{D}$ systems we consider the Hubbard model

$$
\mathcal{H}=\sum_{\mathbf{k} \sigma} \varepsilon_{\mathbf{k} \sigma} c_{\mathbf{k} \sigma}^{+} c_{\mathbf{k} \sigma}+U \sum_{i} n_{i \uparrow} n_{i \downarrow}
$$

with the electronic dispersion

$$
\varepsilon_{\mathbf{k}}=-2 t\left(\cos k_{x}+\cos k_{y}\right)+4 t^{\prime}\left(\cos k_{x} \cos k_{y}+1\right)-\mu
$$

where $t$ and $t^{\prime}$ are the nearest- and next-nearest neighbor hopping parameters, $U$ is the Hubbard on-site repulsion, $n_{i \sigma}=c_{i \sigma}^{+} c_{i \sigma}$.

Let us first consider the noninteracting case. The corresponding plots of the noninteracting density of states for different $t^{\prime}$ are shown in Fig. 1, For $t^{\prime}<0.5 t$ the density of states has logarithmic singularity at the energy $4 t-8 t^{\prime}$ measured from the bottom of the band. For $t^{\prime}=0.5 t$ there are lines of VHS along the $k_{x}=0$ and $k_{y}=0$ directions, and the density of states has stronger divergence $\rho(\varepsilon) \propto 1 / \varepsilon^{1 / 2}$ at the bottom of the band (the so-called flat band case [17, 18]), which is analogous to the giant VHS in 3D systems [19]. The dynamical susceptibility of the noninteracting electronic gas with the dispersion (2) is

$$
\chi_{q}^{0}=-\frac{T}{N} \sum_{k} \mathcal{G}_{k} \mathcal{G}_{k+q}
$$

where $\mathcal{G}_{k}=\left(\mathrm{i} \nu_{n}-\varepsilon_{\mathbf{k}}\right)^{-1}$ is the bare electronic Green function, $k=\left(\mathbf{k} ; \mathrm{i} \nu_{n}\right)$ and $q=\left(\mathbf{q} ; \mathrm{i} \omega_{n}\right)$, $\nu_{n}=(2 n+1) \pi T$ and $\omega_{n}=2 n \pi T$ are the Fermi and Bose Matsubara frequencies. In the presence of VHS the uniform susceptibility is logarithmically divergent near Van-Hove band fillings with $\mu=0$,

$$
\chi_{0}^{0} \simeq \frac{1}{2 \pi t} \ln \frac{t}{\max (|\mu|, T)} .
$$

According to the Stoner criterion, this leads to a possibility of ferromagnetism for $U>$ $U_{c}^{\text {Stoner }}=1 / \chi_{0}^{0}$. However, the momentum dependence $\chi_{(\mathbf{q} ; 0)}^{0}$ at low $T \ll t$ and $\mu \neq 0$ has its maximum at $\mathbf{q} \neq 0$ (see Ref. [11] and Figs. 4/5] with $\Delta=0$ below). In the random phase approximation (RPA)

$$
\chi_{q}^{\mathrm{RPA}}=\frac{\chi_{q}^{0}}{1-U \chi_{q}^{0}} .
$$

this results in an instability of ferromagnetic ground state, since $\chi_{(\mathbf{q} ; 0)}^{\mathrm{RPA}}$ is divergent at $\mathbf{q} \neq 0$ even for $U<U_{c}^{\text {Stoner }}$, which implies the spin-density wave instability in this approach. This shortcoming holds also in the Hertz-Moriya-Millis theory, not considering the renormalization of momentum dependence of the electron-electron interaction and implies the impossibility of ferromagnetism near van Hove fillings in existing spin-fluctuation approaches.

\subsection{The spin-fermion model}

For the purpose of investigation of magnetic properties beyond RPA-type approaches we consider the spin-fermion model [13]

$$
\mathcal{Z}_{\mathrm{sf}}\left[\eta, \eta^{+}\right]=\int D\left[\mathbf{S} ; c, c^{+}\right] \exp (-\beta \mathcal{S}),
$$



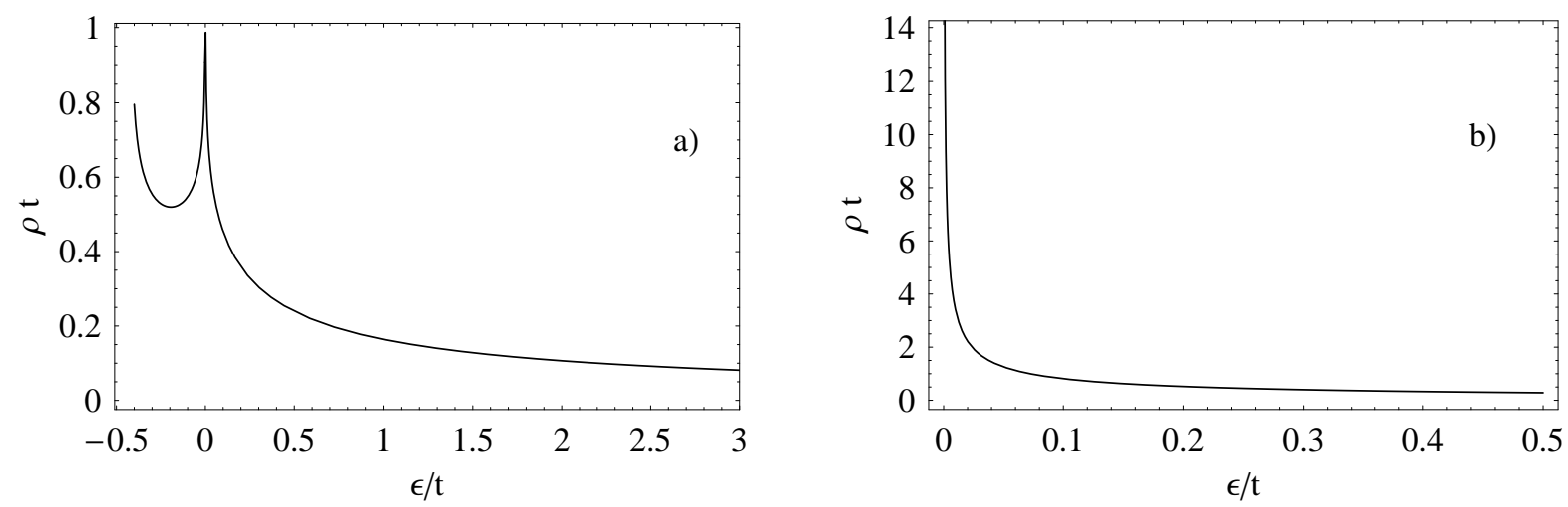

Figure 1: Electronic noninteracting density of states $\rho(\varepsilon)$ at a) $t^{\prime}=0.45 t$, b) $t^{\prime}=0.50 t$

$$
\begin{gathered}
\mathcal{S}=\sum_{k \sigma} c_{k \sigma}^{+}\left(-\mathrm{i} \nu_{n}+\varepsilon_{\mathbf{k}}\right) c_{k \sigma}+\mathcal{S}_{S}+\frac{I}{N} \sum_{q} \mathbf{s}_{q} \mathbf{S}_{-q}-\sum_{k \sigma}\left(\eta_{k \sigma}^{+} c_{k \sigma}+c_{k \sigma}^{+} \eta_{k \sigma}\right), \\
\mathcal{S}_{S}=\frac{1}{N} \sum_{q} \chi_{q}^{-1} \mathbf{S}_{q} \mathbf{S}_{-q} .
\end{gathered}
$$

where the Grassman fields $c_{k \sigma}, c_{k \sigma}^{+}$correspond to electronic degrees of freedom, the field $\mathbf{S}_{q}$ describes the collective spin degrees of freedom, corresponding to paramagnons, $\chi_{q}$ is the "bare" susceptibility of the paramagnon subsystem, $\mathbf{s}_{q}=\sum_{k \sigma \sigma^{\prime}} \bar{c}_{k \sigma}^{+} \vec{\sigma}_{\sigma \sigma^{\prime}} c_{k+q \sigma^{\prime}}$ is the spin operator of itinerant electrons, $\vec{\sigma}$ are the Pauli matrices, $I$ is the interaction of the paramagnon and electron subsystems, $\eta_{k \sigma}, \eta_{k \sigma}^{+}$are the fermionic sources fields, $\beta=1 / T$ is the inverse temperature, and $N$ is the number of sites.

Although the model (6) was previously derived from the Hubbard model via the Hubbard-Stratonovich transformation, this way of derivation meets the problem of the socalled Fritz ambiguity [30, 31, 32, 33, 34, 35] and the resulting action is not uniquely defined. In this paper we use a different way to establish connection between the spin-fermion and Hubbard model, and show that the former describes magnetic degrees of freedom of the latter, see Appendix A. The equivalence with the Hubbard model requires

$$
I=U, \chi_{q}^{-1}=\left(\chi_{q}^{\mathrm{el}}\right)^{-1}+U^{2} H_{q},
$$

where $\chi_{q}^{\mathrm{el}}$ is the exact magnetic susceptibility of the Hubbard model, which is calculated self-consistently in the framework of spin-fermion model according to $\left(\chi_{q}^{\mathrm{el}}\right)^{-1}=H_{q}^{-1}-U, H_{q}$ is the irreducible electronic polarization operator. In the most part of the paper we consider, however, the spin-fermion model with arbitrary $\chi_{q}$ and $I$.

To investigate the magnetic properties of the model (6) it is convenient to rewrite the action in terms of the bosonic fields only [12]. Integrating out electronic degrees of freedom and expanding the resulting functional in the series in $I$ we obtain

$$
\begin{gathered}
\mathcal{Z}_{\mathrm{sf}}\left[\eta, \eta^{+}\right]=\int D[\mathbf{S}] \exp \left[-\beta \mathcal{S}_{S}-\beta \mathcal{S}_{\mathrm{int}}-\sum_{k k^{\prime} \sigma \sigma^{\prime}} \eta_{k \sigma}^{+} \mathbb{G}_{k \sigma, k^{\prime} \sigma^{\prime}} \eta_{k^{\prime} \sigma^{\prime}}\right], \\
\mathcal{S}_{\text {int }}=T \sum_{n=2}^{\infty} \frac{1}{n} \operatorname{Tr}\left[\left(\frac{I}{N} \mathcal{G}_{k} \vec{\sigma} \mathbf{S}_{k-k^{\prime}}\right)^{n}\right]_{k \sigma, k^{\prime} \sigma^{\prime}},
\end{gathered}
$$

where

$$
\mathbb{G}_{k \sigma, k^{\prime} \sigma^{\prime}}[\mathbf{S}]=\left\{\left(\mathrm{i} \omega_{n}-\varepsilon_{\mathbf{k}}\right) \delta_{k k^{\prime}} \delta_{\sigma \sigma^{\prime}}-(I / N) \vec{\sigma}_{\sigma \sigma^{\prime}} \mathbf{S}_{k-k^{\prime}}\right\}_{k \sigma, k^{\prime} \sigma^{\prime}}^{-1}
$$


is the Green function of electrons, propagating in the presence of an external field $\mathbf{S}, \mathcal{S}_{\text {int }}$ corresponds to paramagnon interaction, the exponent $n$ corresponds the power of matrix in the square brackets taken with respect to indices $k \sigma, k^{\prime} \sigma^{\prime}$. Coefficients of the expansion of the interaction $\mathcal{S}_{\text {int }}$ in powers of the spin fields $S_{k-k^{\prime}}^{j}(j=x, y, z)$ determine the vertices of paramagnon interaction

$$
\Gamma_{0}^{j_{1} \ldots j_{r}}\left(q_{1}, \ldots, q_{r}\right)=\frac{T I^{r}}{r} \sum_{\mathcal{P}_{q}} \sum_{k} \operatorname{Tr}_{\sigma}\left[\prod_{i=1}^{r}\left(\mathcal{G}_{k+\sum_{l=1}^{i} q_{l}} \sigma^{j_{i}}\right)\right]
$$

$\left(\mathcal{P}_{q}\right.$ corresponds to all possible permutations of momenta $q_{i}, \operatorname{Tr}_{\sigma}$ is the trace with respect to spin variables). Taking into account the fourth order vertex only leads to an effective $\phi^{4}$ model [8, 9] with the paramagnon dispersion $\left(\chi_{q}^{\mathrm{RPA}}\right)^{-1}$ and the interaction $\Gamma_{0}\left(q_{1}, q_{2}, q_{3}, q_{4}\right)$. Below we consider, however, the effect of infinite sequence of vertices (10) on the magnetic properties.

Diagram technique for the model (8) is considered in Appendix B and contains the following elements:

- the propagators of the longitudinal and transverse paramagnons $\mathcal{R}_{q}$;

- the electronic Green functions $\mathcal{G}_{k}$, connected in loops (10);

- the electron-paramagnon interaction vertices $I \vec{\sigma}$.

These rules are used below to calculate physically observable quantities.

\subsection{Spin susceptibility}

To investigate magnetic properties of the model (6) we consider magnetic susceptibility, defined as the causal double-time Green function

$$
\chi_{q}^{\mathrm{el}}=\frac{1}{4 N} \int_{0}^{\beta} d \tau e^{\mathrm{i} \omega_{n} \tau}\left\langle T_{\tau} s_{\mathbf{q}}^{+}(\tau) s_{-\mathbf{q}}^{-}(0)\right\rangle
$$

and the paramagnon propagator

$$
\mathcal{R}_{q}=\frac{1}{N} \int_{0}^{\beta} d \tau e^{\mathrm{i} \omega_{n} \tau}\left\langle T_{\tau} S_{\mathbf{q}}^{+}(\tau) S_{-\mathbf{q}}^{-}(0)\right\rangle
$$

Here $s_{q}^{ \pm}(\tau)$ and $S_{q}^{ \pm}(\tau)$ are the operators in the Heisenberg representation, corresponding to the $s_{q}^{ \pm}$and $S_{q}^{ \pm}$fields, $T_{\tau}$ denotes the chronological product with respect to the imaginary time. To obtain the result for the susceptibility $\chi_{q}^{\mathrm{el}}$, one can differentiate Eq. (8) with respect to $\eta$. Classifying corresponding contributions to the one particle reducible and irreducible, we obtain (see Appendix A)

$$
\chi_{q}^{\mathrm{el}}=\frac{H_{q}}{1-I^{2} H_{q} \chi_{q}}+\tilde{\chi}_{q}^{\mathrm{el}}
$$

where $\tilde{\chi}_{q}^{\mathrm{el}}$ is the non-paramagnon contribution to the electronic susceptibility $\left(\tilde{\chi}_{q}^{\mathrm{el}}=U H_{q}^{2}\right.$ for the Hubbard model). The result (13) reduces for $H_{q}=\chi_{q}^{0}$ to the RPA result for the spin susceptibility.

The general expression for the paramagnon propagator is

$$
\mathcal{R}_{q}^{-1}=\chi_{q}^{-1}-\Xi_{q}
$$


a)

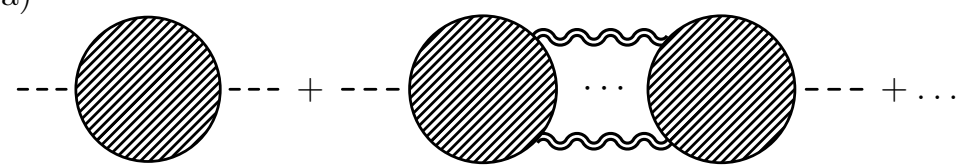

b)

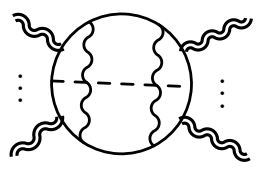

Figure 2: (a) Series of diagrams for the polarization operator of electronic subsystem; the dashed circle represents the sum of all one-loop diagrams, as in Fig. 2b. (b) The example of the one-loop diagram, double wavy lines correspond to the longitudinal or the transverse propagator, denoted by dashed and wavy lines, respectively

where $\Xi_{q}$ is the paramagnon self-energy. It may be established diagrammatically that this self-energy can be expressed through the polarization operator $H_{q}$ according to $\Xi_{q}=I^{2} H_{q}$ (this relation is in fact a consequence of the form of the interaction term of the model (6 6 )).

The polarization operator $H_{q}$ can be represented as a set of diagrams, which contain loops of electronic Green functions (the one-loop vertex functions, defined in Appendix B), connected by two or more paramagnon lines (see Fig. 2). In the present paper we neglect contributions to $H_{q}$, which contain more than one electronic loop (second and next terms in Fig. 2a), since we expect that their contribution to the magnetic susceptibility is small. The contribution of the diagrams with only one electronic loop (Fig. 2b) corresponds the one-loop 2-point vertex

$$
\begin{array}{r}
H_{q}=\left\langle\Pi_{q}[\mathbf{S}]\right\rangle_{0}=:-\frac{1}{2 I^{2}} \Gamma_{1-\text { loop }}^{z z}(q,-q), \\
\Pi_{q}[\mathbf{S}]=-\frac{T}{2 N} \sum_{k_{1} k_{2}} \operatorname{Tr}_{\sigma}\left[\mathbb{G}_{k_{1}, k_{2}}[\mathbf{S}] \sigma^{z} \mathbb{G}_{k_{2}+q, k_{1}+q}[\mathbf{S}] \sigma^{z}\right] .
\end{array}
$$

According to the Mermin-Wagner theorem [21, the long range order in 2D systems is possible at $T=0$ only. The susceptibility (13) in the limit $T \rightarrow 0$ corresponds to the susceptibilities of the ordered state, averaged over directions:

$$
\lim _{T \rightarrow 0} \chi_{q}^{\mathrm{el}}=\frac{1}{3}\left(\chi_{q}^{z z}+\chi_{q}^{+-}\right)_{T=0}^{\mathrm{el}} .
$$

The necessary condition of the ferromagnetic ground state is

$$
I^{2} \chi_{0} H_{q=0}=1 \text { at } T \rightarrow 0
$$

(generalized Stoner criterion), and the positivity of the spectrum of excitations of the static paramagnons $\omega_{\mathbf{q}} \equiv \mathcal{R}_{(\mathbf{q} ; 0)}^{-1}$ at $T \rightarrow 0$, which is fulfilled if the product $\chi_{(\mathbf{q} ; 0)} H_{(\mathbf{q}, 0)}$ is maximal at $\mathbf{q}=0$. This criterion is violated in RPA, where $\omega_{\mathbf{q}}$ is not positively defined in the $T \rightarrow 0$ limit in the lowest (second) order of perturbation theory in $I$ (see Section 2.1). Moreover, the corresponding energy of the paramagnon interaction $\Gamma_{0}(0,0,0,0)=-12 I^{4} \rho^{\prime \prime}(\mu)$ is also negative near VHS. To investigate the possibility of ferromagnetism we consider below the results for the polarization operator $H_{q}$ beyond RPA.

\subsection{The static and the quantum contributions. The static approx- imation}

In the assumption of ferromagnetically ordered ground state considered expressions can be simplified in the $T \rightarrow 0$ limit analogously to the earlier investigated case of antiferromagnetic order [14, 15, 16]. 


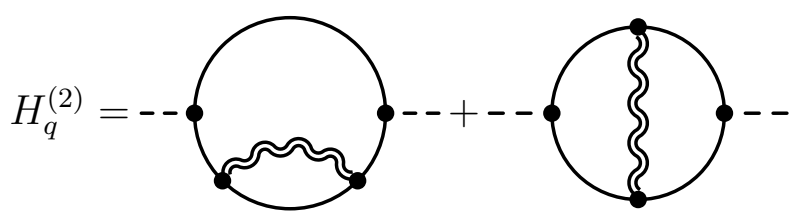

Figure 3: The diagrams for the polarization operator $H_{q}$ in the lowest order in $I$, the notations are the same as in Fig. 2

Let us consider for example the lowest (second) order result of perturbation theory in $I$ for the polarization operator susceptibility $H_{q}$. There are two topologically different diagrams in this order, see Fig. 3. The corresponding analytical expression reads (cf. Appendix B)

$$
H_{q}^{(2)}=-\frac{6 T^{2} I^{2}}{N^{2}} \sum_{k q^{\prime}} \mathcal{G}_{k}^{2} \mathcal{G}_{k+q} \mathcal{G}_{k+q^{\prime}} \mathcal{R}_{q^{\prime}}+\frac{T^{2} I^{2}}{N^{2}} \sum_{k q^{\prime}} \mathcal{G}_{k} \mathcal{G}_{k+q} \mathcal{G}_{k+q^{\prime}} \mathcal{G}_{k+q+q^{\prime}} \mathcal{R}_{q^{\prime}},
$$

We assume that the paramagnon propagator $\mathcal{R}_{q}$ at small momenta and frequencies for the Fermi level lying near VHS has a form (see, e.g., Ref. [22])

$$
\mathcal{R}_{\mathbf{q}, \mathrm{i} \omega_{n}}=\frac{A}{\mathbf{q}^{2}+\xi^{-2}+B\left|\omega_{n}\right| / \max \left(t q_{+} q_{-},\left|\omega_{n}\right|\right)}+\mathcal{R}_{\mathbf{q}, \mathrm{i} \omega_{n}}^{\mathrm{r}},
$$

where $A, B>0$ are some constants, $\xi$ is the correlation length of spin fluctuations, $\mathcal{R}_{\mathbf{q}, i \omega_{n}}^{\mathrm{r}}$ is the contribution, which is regular at $q \rightarrow 0, \xi \rightarrow \infty$ and neglected below. The result (19) can be obtained from the general formula (14) under the assumption that the expansion of the polarization operator $H_{q}$ in the momentum and frequency has the same form as one for the noninteracting susceptibility $\chi_{q}^{0}$, the effect of the interaction is in the change of the parameters $A$ and $B$ only. While the condition $B>0$ being a consequence of the analytical properties of the susceptibility as a function of frequency, the positivity of the constant $A$ is analyzed below in the section 3.1.

It follows from the Eq. (19) that the dominant contribution to the momenta- and frequency sum in Eq. (18) comes from the region with $|\mathbf{q}| \sim \xi^{-1}$ and the zeroth Matsubara frequency. If the condition

$$
(t / T)^{1 / 2} \ll \xi
$$

is fulfilled the contribution of the terms with nonzero Matsubara frequency in the sum over $\omega^{\prime}$ in the Eq. (19) can be neglected, together with the q-dependence of the electronic Green function. The condition (20) is certainly fulfilled in 2D case at finite $T$ above the ordered ground state, since of the correlation length is exponentially large at small $T$ (cf., for example, Refs. [23, 24]). This differs present theory from the earlier considered 3D case [12], where above-discussed approximations give only qualitatively, but not quantitatively correct description of magnetic properties.

Within these approximations the sum (18) takes a form

$$
H_{q}^{(2)}=\frac{T^{2} I^{2}}{N^{2}} \sum_{k}\left(-6 \mathcal{G}_{k}^{3} \mathcal{G}_{k+q}+\mathcal{G}_{k}^{2} \mathcal{G}_{k+q}^{2}\right) \sum_{\mathbf{q}^{\prime}} \frac{1}{\omega_{\mathbf{q}^{\prime}}} .
$$

Analogous approximations are also applicable to higher order diagrams. The results coincide with those for the model with the action

$$
\mathcal{Z}_{\xi \rightarrow \infty}\left[\eta, \eta^{+}\right]=\int d^{3} S \exp \left[-\frac{3 I^{2} S^{2}}{2 \Delta^{2}}-\beta \sum_{k} \eta_{k}^{+}\left(\mathrm{i} \nu_{n}-\varepsilon_{\mathbf{k}}-I \vec{\sigma} \mathbf{S}\right)^{-1} \eta_{k}\right],
$$


which contains only one uniform static mode $\mathbf{S}$ with the propagator

$$
\frac{1}{3}(\Delta / I)^{2} \equiv \frac{T}{N} \sum_{\mathbf{q}} \frac{1}{\omega_{\mathbf{q}}}
$$

The Green functions of Bose and Fermi fields of the model (22) can be expressed in closed analytical form, cf. [15, 16]. The static approximation and the neglection of qdependence of electronic Green function leads to zero momentum- and frequency transfer along the paramagnon lines in all diagrams; hence, $\mathbb{G}_{k k^{\prime}}[\mathbf{S}]$ becomes diagonal with respect to momenta and frequency:

$$
\mathbb{G}_{k k^{\prime}}[\mathbf{S}] \rightarrow G_{k}(\mathbf{S}) \delta_{k k^{\prime}}, G_{k}(\mathbf{S})=\left(\mathrm{i} \omega_{n}-\varepsilon_{\mathbf{k}}-I \vec{\sigma} \mathbf{S}\right)^{-1}
$$

Therefore we obtain

$$
H_{q}=\left\langle\Pi_{q}(\mathbf{S})\right\rangle_{\xi \rightarrow \infty}
$$

where

$$
\Pi_{q}(\mathbf{S})=-\frac{T}{2 N} \sum_{k} \operatorname{Tr}_{\sigma}\left[G_{k}(\mathbf{S}) \sigma^{z} G_{k+q}(\mathbf{S}) \sigma^{z}\right],
$$

the subscript $\xi \rightarrow \infty$ corresponds to averaging with the functional (22). Calculation of the average in the Eq. (25) leads to

$$
H_{q}=\int d^{3} \mathbf{S}\left[\frac{1}{3} \Pi_{\|}\left(\mathbf{q} \mid S \operatorname{sign} S^{z}\right)+\frac{2}{3} \Pi_{\perp}\left(\mathbf{q} \mid S \operatorname{sign} S^{z}\right)\right] \exp \left(-\frac{3 I^{2} S^{2}}{2 \Delta^{2}}\right)
$$

where

$$
\Pi_{\|, \perp}(\mathbf{q} \mid S)=-\frac{1}{N} \sum_{\mathbf{k}} \frac{f\left(\varepsilon_{\mathbf{k}}-I S\right)-f\left(\varepsilon_{\mathbf{k}+\mathbf{q}} \mp I S\right)}{\varepsilon_{\mathbf{k}}-\varepsilon_{\mathbf{k}+\mathbf{q}}-I S \pm I S} .
$$

Similarly we find the electronic Green function:

$$
G_{k}=\frac{\delta^{2} \mathcal{Z}_{\mathrm{sf}}\left[\eta, \eta^{+}\right]}{\delta \bar{\eta}_{k}^{+} \delta \eta_{k}}=\left\langle G_{k}(\mathbf{S})\right\rangle_{\xi \rightarrow \infty} .
$$

The explicit expression for $G_{k}$ was obtained earlier in the paper [16]. The corresponding spectral function

$$
A(\mathbf{k}, \omega)=-\frac{1}{\pi} \operatorname{Im} G_{\mathbf{k}}(\omega)=\frac{9}{\sqrt{6 \pi} \Delta^{3}}\left(\omega-\epsilon_{\mathbf{k}}\right)^{2} \exp \left[-\frac{3\left(\omega-\epsilon_{\mathbf{k}}\right)^{2}}{2 \Delta^{2}}\right]
$$

has two-peak (non-quasiparticle) structure at the Fermi surface, which destroys the quasiparticle picture due to strong ferromagnetic fluctuations (see Fig. 10 below). As it was discussed in the paper [24], the corresponding violation of Fermi-liquid behavior corresponds to a quasi-splitting of the Fermi surface at low temperatures, which is related to the change of the the electronic spectrum in the vicinity of the magnetically ordered ground state.

\section{The Results for the Phase Diagram, Paramagnon Vertices and Spectral Functions}

\subsection{The irreducible susceptibility}

To investigate the possibility of the ferromagnetic order in the ground state we consider the momentum dependence of the irreducible static susceptibility (polarization operator) $H_{(\mathbf{q}, 0)}$ 

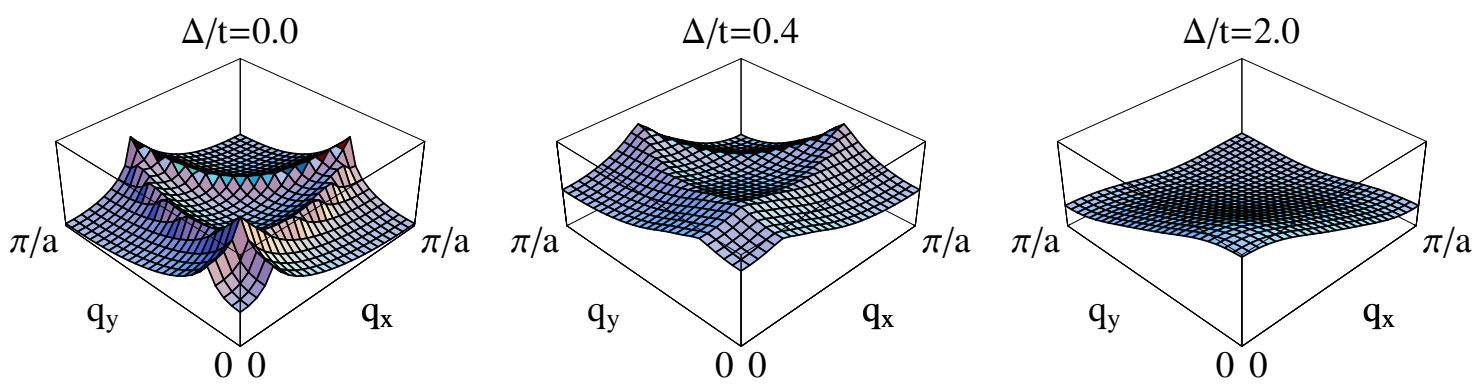

Figure 4: Momentum dependence of the static irreducible electronic susceptibility $H_{\mathbf{q}}$ in the first quarter of the Brillouin zone for $t^{\prime} / t=0.45, n=0.583>n_{\mathrm{VH}}=0.466$ and different values of $\Delta$
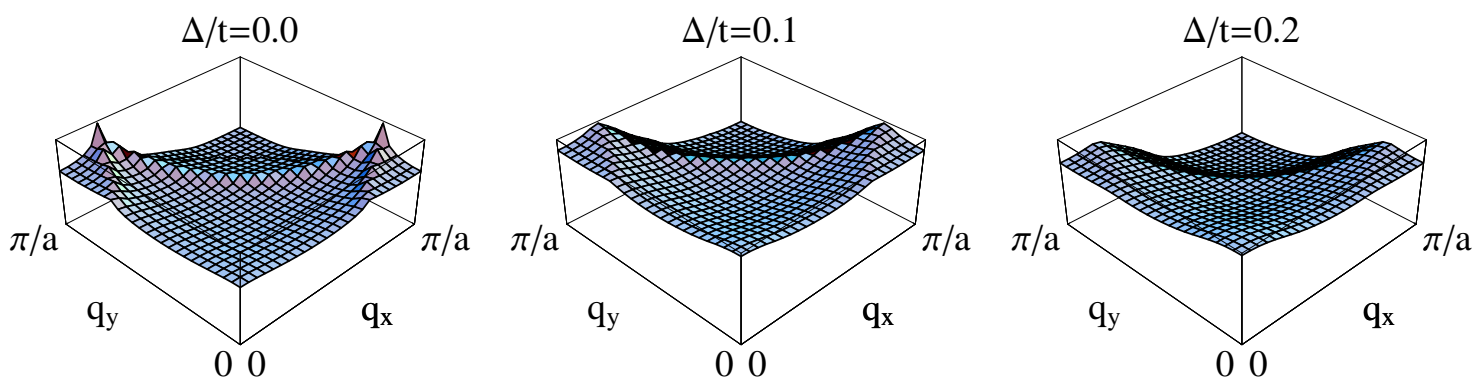

Figure 5: The same as in Fig. 4 for $n=0.338<n_{\mathrm{VH}}$

at $T \rightarrow 0$. The plots of $H_{(\mathbf{q}, 0)}$, calculated according to the Eq. (25) in a quarter of the Brillouin zone for $t^{\prime}=0.45 t$ and different values of $n, \Delta$ are presented in Figs. 4, 5. The chemical potential $\mu$ is adjusted to keep the number of electrons $n=(2 / N) \sum_{\mathbf{k}} \int_{-\infty}^{\mu} d \varepsilon A(\mathbf{k}, \varepsilon)$ equal to the noninteracting value with increasing $\Delta$, the spectral function $A(\mathbf{k}, \varepsilon)$ is determined by the Eq. (29).

For $\Delta=0$ (which corresponds to $I=0$ ) the global maximum of the static susceptibility is located at the point $\mathbf{q} \neq 0$, so that for both the positions of the Fermi level above and below the VHS the condition of the positivity of paramagnon excitation spectrum is violated. With increasing $\Delta$ the static irreducible susceptibility has a maximum at $\mathbf{q}=0$ : for $n<n_{\mathrm{VH}}$ the local maximum at $\mathbf{q}=0$ becomes the global one and for $n>n_{\mathrm{VH}}$ local maximum shifts to the point $\mathbf{q}=0$. Therefore, in both cases the interaction of electrons with paramagnons leads to the global maximum of polarization operator of the electronic subsystem at $\mathbf{q}=0$, which corresponds to a possibility of the ferromagnetically ordered ground state.

To determine the critical value of $\Delta$ (the minimal value of $\Delta$, at which the ferromagnetic ground state is possible) we consider the second derivative of the irreducible susceptibility with respect to $q_{x}$ (or $q_{y}$ ) $\partial_{q_{x}}^{2} H_{\mathbf{q}} \equiv \partial^{2} H_{\mathbf{q}} / \partial q_{x}^{2}$ at the point $\mathbf{q}=0$ (cf. Appendix $\mathrm{C}$ ). The results of calculation of this are presented in Fig. 6. Changing of sign of the second derivative determines the critical values $\Delta_{c} \sim t$, which depend on the electronic concentration. 


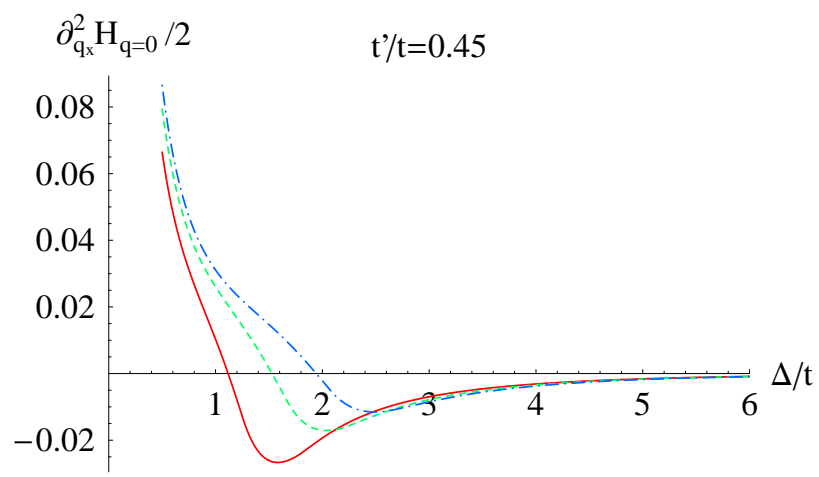

Figure 6: The derivative $\partial_{q_{x}}^{2} H_{\mathbf{q}=0}$ vs $\Delta$ for: $n=0.533$ - solid line, $n=0.583$ - dashed line, $n=0.626$ dash-dotted line
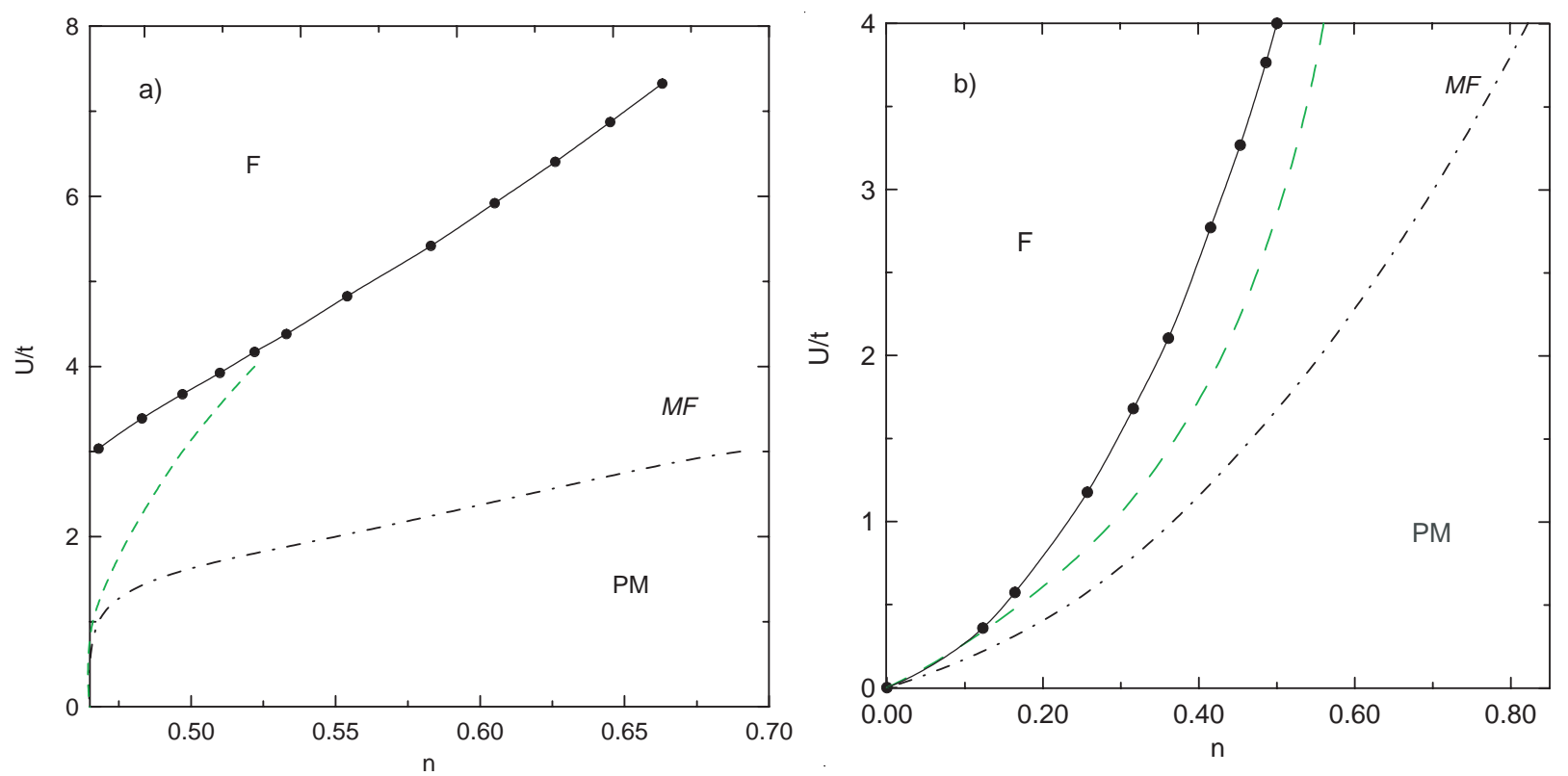

Figure 7: Phase diagram in $n-U$ plane: (a) $t^{\prime} / t=0.45$; (b) $t^{\prime} / t=0.50$. Solid line - the result of the present paper; dashed line - the results of functional renormalization approach methods [17]; dash-dotted line - the result of the mean field theory 


\subsection{The phase diagram of the Hubbard model}

To obtain the phase diagram it is necessary to establish the relation between the critical value of parameter $\Delta_{c}$ and the corresponding spin-fermion interaction $I_{c}$ or the Hubbard interaction $U_{c}$. These relations can be found using the generalized Stoner criterion (17). Below we consider the Hubbard model, for which we find $U_{c}=1 / H_{\mathbf{0}}\left(\Delta_{c}\right)$, where $H_{\mathbf{0}}$ is the uniform static polarizaton operator,

$$
H_{\mathbf{0}}=\frac{1}{2 \Delta} \int_{-4+8 t^{\prime}}^{4+8 t^{\prime}} \rho(\varepsilon) \varphi\left(\frac{\varepsilon-\mu}{\Delta}\right) d \varepsilon
$$

$\varphi(x)=\left(3 x^{2}+2\right) \exp \left(-3 x^{2} / 2\right) / \sqrt{6 \pi}$.

The resulting phase diagram in the $n-U$ plane for $t^{\prime} / t=0.45$ and $n>n_{\mathrm{VH}}$ is presented in Fig. $7 \mathrm{a}$ (the case $n<n_{\mathrm{VH}}$ is not considered in the following, because the incommensurate spin fluctuations with the wavevector $\mathbf{Q}$ far from the point $\mathbf{q}=0$ are not taken into account in the present approach). The critical values $U_{c}$ for $n>n_{\mathrm{VH}}$ are larger than the corresponding mean-field values $U_{c}^{\mathrm{MF}}$. The results for $n$ far from $n_{\mathrm{VH}}$ are in qualitative agreement with the functional renormalization group (fRG) results for the Hubbard model [17]. However, in contrast to the fRG results the critical value $U_{c}$ is nonzero for $n=n_{\mathrm{VH}}$, which is related to the breakdown of the quasiparticle picture of the electronic spectrum owing to the ferromagnetic fluctuations. Thus, the non-Fermi-liquid properties of the electronic spectrum in $2 \mathrm{D}$ systems become important for the criterion of the ferromagnetism in the vicinity of the VHS.

The phase diagram for the flat band case $t^{\prime} / t=0.50$ is presented in Fig. 7b. In this case the critical values $U_{c}$ are also larger than the mean field values $U_{c}^{\mathrm{MF}}$, and there is qualitative agreement between the fRG and obtained here results for all values of electronic concentration $n$ [17].

\subsection{The paramagnon interaction vertex}

Let us consider the vertex $\Gamma^{j j j^{\prime} j^{\prime}}\left(q_{1}, q_{2}, q_{3}, q_{4}\right)$, which determines the energy of the paramagnon interaction and defined by the Eq. (47) of Appendix B. Below we are interested in $j \neq j^{\prime}$-the component of this vertex. This component can be expressed through the corresponding 2-particle irreducible vertex $\Gamma_{\mathrm{irr}}^{j j j^{\prime} j^{\prime}}\left(q_{1}, q_{2}, q_{3}, q_{4}\right)$, which is defined such that it cannot be split into two connected parts by removing two paramagnons lines. In general the relation between these two vertices is determined by the parquet set of diagrams.

To simplify the relation between the vertices we generalize the model (66) introducing the $M$-component spin field $\mathbf{S}$ (cf. Refs. [25, 26]), $j=1, \ldots, M$. Due to internal sums over spin indices, the dominant contribution into the full vertex in the limit $M \rightarrow \infty$ comes from the ladder set of diagrams with equal spin indices of internal paramagnon Green functions (see Fig. 8).

This allows to obtain a simple relation between the full and irreducible vertex, $\Gamma^{j j j^{\prime} j^{\prime}}$ and $\Gamma_{\mathrm{irr}}^{j j j^{\prime} j^{\prime}}$ to first order in $1 / M$

$$
\begin{gathered}
\Gamma^{j j j^{\prime} j^{\prime}}\left(q_{1}, q_{2}, q_{3}, q_{4}\right)=\Gamma_{\mathrm{irr}}^{j j j^{\prime} j^{\prime}}\left(q_{1}, q_{2}, q_{3}, q_{4}\right) \\
-\frac{T}{8 N} \sum_{j^{\prime \prime}, p_{1}, p^{\prime}} \Gamma^{j j j^{\prime \prime} j^{\prime \prime}}\left(q_{1}, q_{2}, p, p^{\prime}\right) \mathcal{R}_{p} \mathcal{R}_{p^{\prime}} \Gamma_{\mathrm{irr}}^{j^{\prime \prime} j^{\prime \prime} j^{\prime} j^{\prime}}\left(-p,-p^{\prime}, q_{3}, q_{4}\right) \delta_{q_{1}+q_{2},-p-p^{\prime}}
\end{gathered}
$$




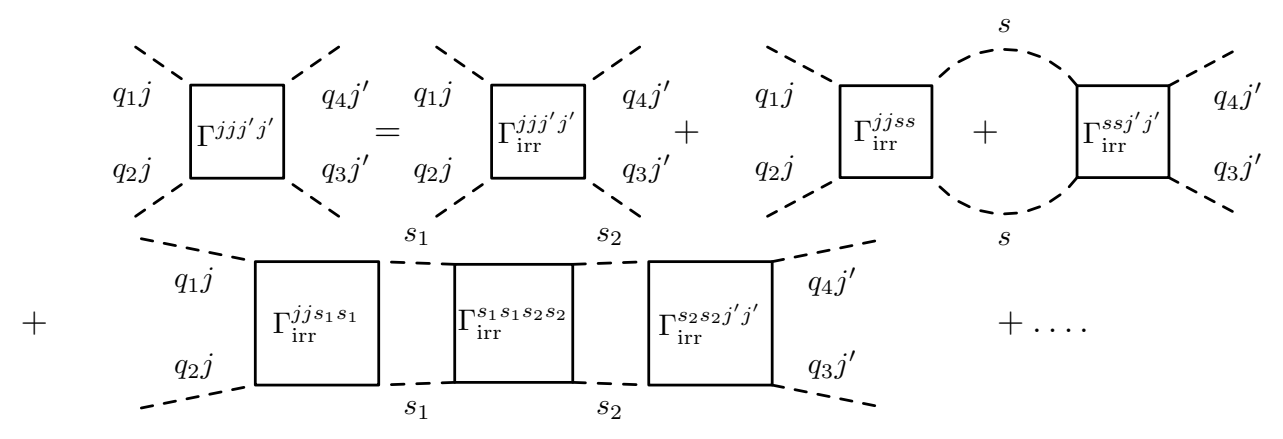

Figure 8: The ladder diagrams for the paramagnon vertex

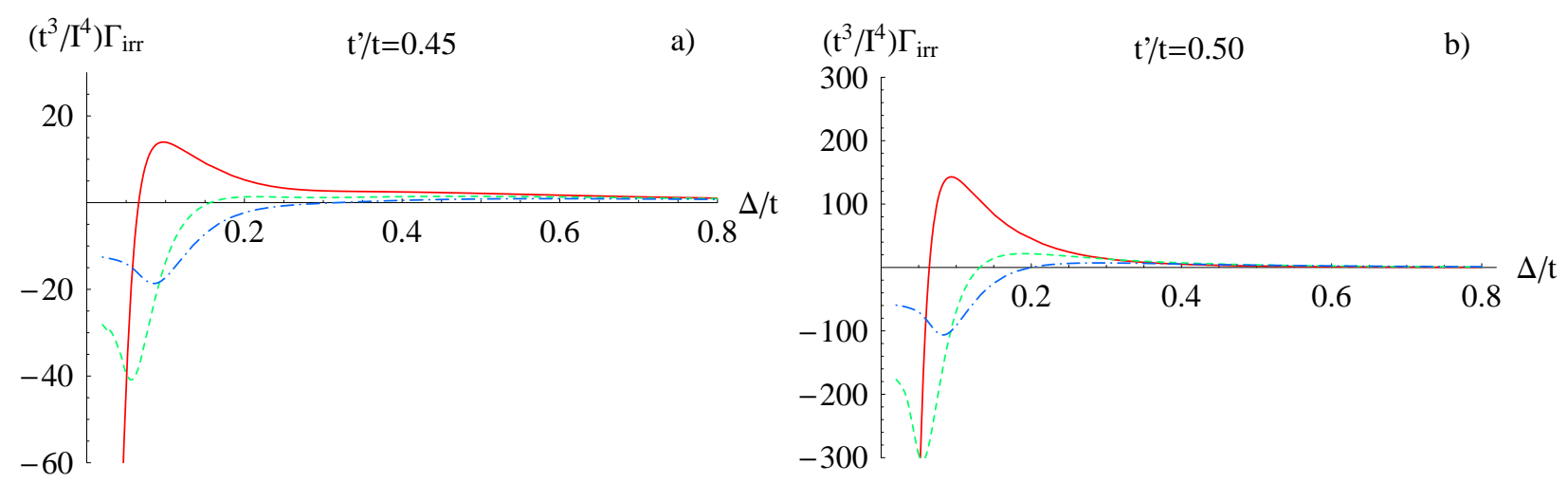

Figure 9: The irreducible vertex $\left(t^{3} / I^{4}\right) \Gamma_{\text {irr }}$ vs $\Delta$ at different electronic concentration $n$ and $t^{\prime}:$ (a) $t^{\prime}=0.45 t$ : $n=0.533$ - solid line, $n=0.583-$ dashed line, $n=0.626$ - dot-dashed line; (b) $t^{\prime}=0.5 t: n=0.316-$ solid line, $n=0.415$ - dashed line, $n=0.486-$ dot-dashed line

At large values of the correlation length $\xi$ the dominant contribution to the sum (31) comes from the small momenta $p, p^{\prime}$. Supposing that the external momenta $q_{1}, q_{2}, q_{3}, q_{4}$ are also small and $\Gamma_{\text {irr }}^{j j j^{\prime} j^{\prime}}\left(q_{1}, q_{2}, q_{3}, q_{4}\right)$ is constant in the vicinity of $\mathbf{q}_{1}=\mathbf{q}_{2}=\mathbf{q}_{3}=\mathbf{q}_{4}=0$ (below we denote this constant as $\Gamma_{\text {irr }}$ ) we obtain in the framework of the quasistatic approach

$$
\Gamma^{j j j^{\prime} j^{\prime}}\left(q_{1}, q_{2}, q_{3}, q_{4}\right)=\frac{\Gamma_{\text {irr }}}{1+\Gamma_{\text {irr }} M \zeta_{\mathbf{q}_{1}+\mathbf{q}_{2}} / 2} \delta_{\omega_{1}, 0} \delta_{\omega_{2}, 0} \delta_{\omega_{3}, 0} \delta_{\omega_{4}, 0},
$$

where

$$
\zeta_{\mathbf{q}}=\frac{T}{4 N} \sum_{\mathbf{p}} \mathcal{R}_{\mathbf{p}} \mathcal{R}_{\mathbf{q}-\mathbf{p}}=\frac{T A^{2}}{4 \pi|\mathbf{q}| \sqrt{\mathbf{q}^{2}+4 \xi^{-2}}} \ln \frac{|\mathbf{q}|+\sqrt{\mathbf{q}^{2}+4 \xi^{-2}}}{2 \xi^{-1}} .
$$

Note, that in the limit $\xi \rightarrow \infty$ the quantity $\zeta_{\mathbf{q}}=T A^{2} /\left(4 \pi|\mathbf{q}|^{2}\right) \ln (|\mathbf{q}| \xi)$ diverges at $|\mathbf{q}| \rightarrow 0$, which results in vanishing of the full vertex of paramagnon interaction (32) at $\mathbf{q}_{1}=\mathbf{q}_{2}=$ $\mathbf{q}_{3}=\mathbf{q}_{4}=0$ in accordance with the assumption of existing of long-range order in the ground state 1 .

One can see from the Eq. (32), that the sign of $\Gamma_{\text {irr }}$ at small external momenta is crucial for the possibility of the FM gilound state. If $\Gamma_{\text {irr }}<0$, the expression (32) shows that paramagnons can form bound states, and hence the ferromagnetism is not possible.

\footnotetext{
${ }^{1}$ Actually this vanishing of the vertex generalizes the Adler principle [27] for the electronic system interacting with paramagnons; this is similar to vanishing of the magnon-interaction vertex at $q_{i}=0$ in Heisenberg magnets which was discussed earlier [28].
} 
Let us consider the sign of $\Gamma_{\text {irr }}$ within the quasistatic approach depending on the magnitude of the electron-paramagnon interaction. In general, the vertex $\Gamma_{\text {irr }}$ can be represented diagrammatically as a sum of the contributions with the one-loop vertices $\Gamma_{1-\text { loop }}$, connected by three or more paramagnon lines. Using the same approximation as for the one-particle polarization operator $\Gamma_{\text {irr }}=\Gamma_{1-\text { loop }}$, i. e. neglecting contributions to the irreducible vertex with the number of loops $n>1$ we obtain in the $M=3$ case

$$
\begin{gathered}
\Gamma^{\mathrm{irr}}=\frac{T I^{4}}{4 N} \operatorname{Tr}_{\sigma}\left\langle\sum_{k} G_{k}(\mathbf{S}) \sigma^{z} G_{k}(\mathbf{S}) \sigma^{x} G_{k}(\mathbf{S}) \sigma^{z} G_{k}(\mathbf{S}) \sigma^{x}\right\rangle_{\xi \rightarrow \infty} \\
=\frac{1}{\Delta^{3}} \int_{-4+8 t^{\prime}}^{4+8 t^{\prime}} \rho(\varepsilon) g\left(\frac{\varepsilon-\mu}{\Delta}\right) d \varepsilon, \quad g(x)=\frac{1}{15}\left(2+3 x^{2}-9 x^{4}\right) \exp \left(-3 x^{2} / 2\right) .
\end{gathered}
$$

The results of the numerical calculation of the irreducible vertex in the $T \rightarrow 0$ limit using the Eq. (34) are presented in Fig. 9. One can see that the irreducible vertex $\Gamma_{\text {irr }}$ changes its sign at the values of $\Delta_{c}$, which are much smaller than the values of $\Delta_{c}$, obtained in Section 3.1, and hence they do not result in additional limitations on the possibility of the ferromagnetic ground state.

For $\Gamma_{\text {irr }}>0$ and small momenta $q_{1}, \ldots, q_{4}$ one can neglect the unity in the denominator of (32) in the limit $M \rightarrow \infty$ to obtain

$$
\Gamma^{j j j^{\prime} j^{\prime}}\left(q_{1}, q_{2}, q_{3}, q_{4}\right)=\frac{2}{M \zeta_{\mathbf{q}_{1}+\mathbf{q}_{2}}} \delta_{\omega_{1}, 0} \delta_{\omega_{2}, 0} \delta_{\omega_{3}, 0} \delta_{\omega_{4}, 0}
$$

Note, that for $\Gamma_{\text {irr }} \ll 1$ (i.e. in regime of weak coupling and/or smooth enough density of states) the formula (35) is correct in the considered limit of low temperature $(\xi \rightarrow \infty)$. For higher temperature the unity in the denominator (32) has to be retained also in the limit of large $M$, since $\Gamma_{\text {irr }} \zeta_{0} \ll 1$.

\subsection{The electronic self-energy and spectral functions}

The first order $1 / M$ result (35) for the paramagnon vertex can be used to investigate the influence of the paramagnon interaction on the electronic self-energy $\Sigma$ and other properties of the electronic system. In the framework of the $1 / M$-expansion it was shown [26] that the electronic properties of the model (6) to first order in $1 / M$ are determined by the self-energy $\Sigma_{k}$ and one-particle irreducible vertices of electron-paramagnon interaction $\gamma_{k} \gamma_{k}^{z z}, \gamma_{k}^{z z \perp}$. In the limit $\xi \rightarrow \infty$ these quantities can be obtained from the system of the algebraic equations

$$
\begin{gathered}
\Sigma_{k}=\Delta^{2} \gamma_{k} G_{k}, \\
\gamma_{k}=1+\left(\gamma_{k}^{z z} G_{k}-\gamma_{k}^{2} G_{k}^{2}\right) \Delta^{2} / 3, \\
\gamma_{k}^{z z}=\Delta^{2}\left(2 \gamma_{k}^{3} G_{k}^{3}+\gamma_{k} \gamma_{k}^{z z} G_{k}^{2}+\gamma_{k}^{z z \perp} G_{k}\right)+\alpha \gamma_{k} G_{k}, \\
\gamma_{k}^{z z \perp}=-2 \Delta^{2}\left(\gamma_{k}^{4} G_{k}^{4}+\gamma_{k}^{2} \gamma_{k}^{z z} G_{k}^{3}+\gamma_{k} \gamma_{k}^{z z \perp} G_{k}^{2}\right)-\alpha \gamma_{k}^{2} G_{k}^{2} .
\end{gathered}
$$

where $\alpha=M \Gamma^{j j j^{\prime} j^{\prime}}(0,0,0,0) \zeta_{\mathbf{0}}$. The results of the solution of these equations with account of paramagnon interaction $(\alpha=2$ from $(\underline{35})$ ) and without it $(\alpha=0)$ are presented in Figs. 6-8.

As well as in the absence of the paramagnon interaction [16], the real part of the self-energy has an infinite slope at the Fermi level, and imaginary part has a $\delta$-function 


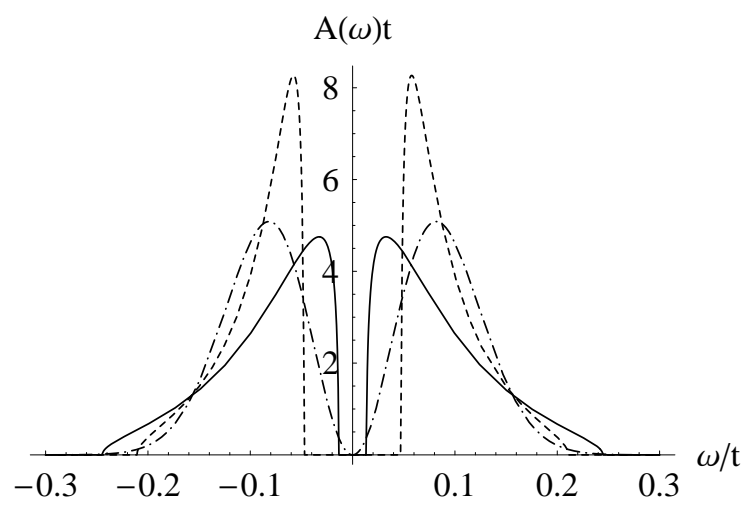

Figure 10: The spectral density $A=-\frac{1}{\pi} \operatorname{Im} G$ vs $\omega \equiv \nu-\varepsilon_{\mathbf{k}}+\mu$, calculated from (36) without ( $\alpha=0$, dashed line) and with account of ( $\alpha=2$, solid line) the paramagnon interaction; dash-dotted line - the same in the quasistatic approach (the formula (29) $), \Delta=0.1 t$
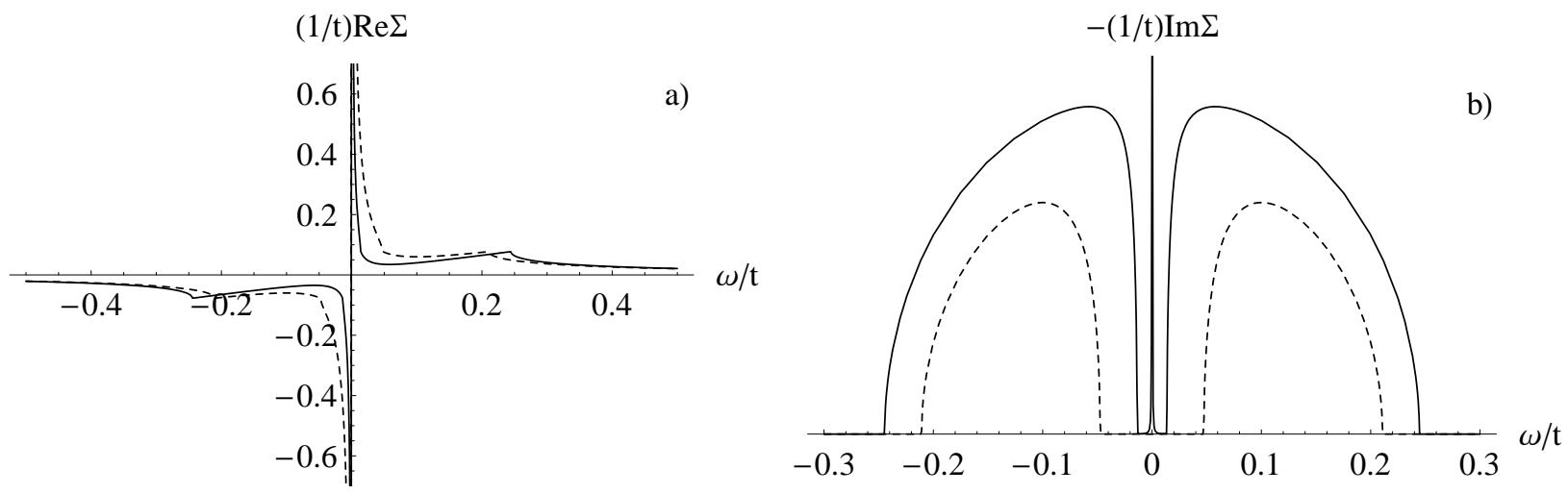

Figure 11: The real (a) and imaginary (b) part of the electronic self-energy $\Sigma(\omega)$, the notations and the parameters are the same as in Fig. 10

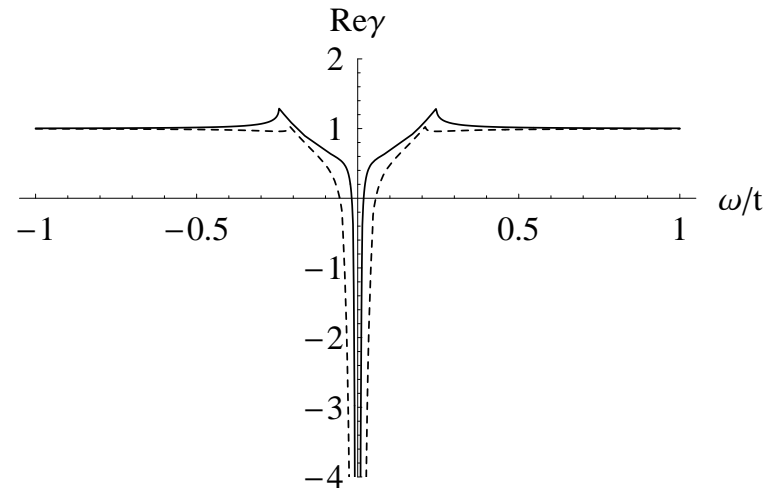

Figure 12: The real part of the electron paramagnon-vertex $\gamma(\omega)$, the notations and the parameters are the same as in Figs. 1011 
peak. It was pointed out above that these anomalies originate from the electron-paramagnon interaction and violate the quasiparticle picture in the vicinity of the Fermi level. Physically this corresponds to appearance of the new quasi-split-Fermi-surfaces shifted from the Fermi level by $\pm \Delta$ in the presence of strong ferromagnetic fluctuations [24]. Note, that existence of the window where the spectral weight is zero is an artefact of $1 / M$-expansion: the comparison with the results of the quasistatic approach shows (Fig. 10) that the spectral weight in this window is reduced, but is not zero. In the presence of the paramagnon interaction the spectrum is less coherent than without it ( $\operatorname{Im} \Sigma$ is enhanced, $A(\omega)$ is smeared), the distance between the peaks of the spectral density $A$ is being reduced. Thus, account of the interaction is important for the description of the electronic spectrum.

\section{Conclusions}

We have considered the necessary conditions of the existence of ferromagnetism in 2D systems in the presence of VHS: the maximum of the polarization operator at $\mathbf{q}=0$, which ensures positive definiteness of the magnetic excitation spectrum and the repulsion between the paramagnons (the latter guarantees the impossibility of the bound state formation).

For the Fermi level position in the vicinity of VHS and in the absence of the electron-paramagnon interaction $I$ these conditions are violated: the momentum dependence of spin susceptibility has its maximum at the wave vector $\mathbf{q} \neq 0$, which corresponds to possibility of the spin density wave in the ground state and the paramagnon interaction is attractive. However, with increasing $I$ the static spin susceptibility acquires a maximum at $\mathbf{q}=0$ and the paramagnon interaction changes its sign. The critical value of the electronparamagnon interaction $I_{c}$ (or the corresponding Hubbard interaction $U_{c}$ ) for which the ferromagnetic ground state is possible exceeds substantially the corresponding value $I_{c}^{\mathrm{MF}}$ $\left(U_{c}^{\mathrm{MF}}\right)$, determined from the Stoner theory, and agrees with the functional renormalization group investigations of Hubbard model for the electronic concentration being in the vicinity of the Van Hove filling. The critical values $I_{c}$ and $U_{c}$ are however finite at the Van Hove electronic filling for $t^{\prime}<0.5 t$, which is related to the non-quasiparticle picture of the electronic spectrum (quasi-splitting of the Fermi surface).

In contrast to the Moriya theory, where the difference of the conditions of ferromagnetism from the Stoner criterion originates from the quantum contributions to the uniform irreducible susceptibility, in the present approach it results from the change of the momentum dependence of the susceptibility by classical spin fluctuations. The change of the uniform irreducible susceptibility by the interaction is also taken into account in the present approach, but it is less important. The kind of the transition from the ferromagnetic to the paramagnetic phase is an open question. One of the possibilities is the existence of intermediate state with the strong short-range order, characterized by the wave vector $\mathbf{Q} \neq 0$. To describe magnetic properties of this phase, it is necessary to consider the excitations with incommensurate wave vector $\mathbf{Q}$ and account for quantum fluctuations, which is a problem for further investigations. The problem of ferromagnetism formation at $n<n_{\mathrm{VH}}$ requires also further consideration.

Besides considered reasons of deviations from the Stoner criterion, the effect of screening due to particle-particle scattering [29] may be important in the Hubbard model. The agreement of the obtained results with the renormalization group results for this model at fillings away from the the Van Hove filling shows, however, that the change of momentum dependence of susceptibility seemingly plays a dominant role. On the other hand, the importance of the non-quasiparticle spectrum of excitations in the vicinity of ferromagnetic 
instability, which has not been taken into account by previous fRG considerations, is demonstrated in the framework of our approach.

The obtained results may serve as a basis for further investigations of itinerant systems in the vicinity of the ferromagnetic instability in the presence of VHS in the electronic spectrum. More detailed investigations of the vicinity of the quantum phase transition and the role of nonanalytic corrections in the system with presence of VHS are needed. It is also of interest to investigate a possibility of the triplet pairing in systems with of VHS in the electronic spectrum.

\section{Acknowledgements}

We are grateful to M. I. Katsnelson for valuable discussions. The work was partially supported by the Project of Scientific Schools of the Russian Basic Research Foundation 4640.2006.2, 07-02-01264-a and 05-02-217704.

\section{Appendix A. The Derivation of the Spin-Fermion Model}

In this Appendix we consider the derivation of the spin-fermion model starting from the Hubbard Hamiltonian (1).

The originally proposed way of derivation of this model [8, 12] was to use the decomposition of the interaction term into charge and spin degrees of freedom, e.g.

$$
U n_{i \uparrow} n_{i \downarrow}=\frac{U}{4}\left[\left(c_{i \sigma}^{+} c_{i \sigma}\right)^{2} \delta_{\sigma \sigma^{\prime}}-\left(c_{i \sigma}^{+} \sigma_{\sigma \sigma^{\prime}}^{x, y, z} c_{i \sigma^{\prime}}\right)^{2}\right]
$$

(the summation over repeated spin indices is assumed) with the subsequent decoupling of quartic terms of fermionic operators using auxiliary scalar or vector fields. The representation (37) is, however, not unique, in particular another, $S U(2)$ symmetric representation,

$$
U n_{i \uparrow} n_{i \downarrow}=\frac{U}{4}\left[\left(c_{i \sigma}^{+} c_{i \sigma}\right)^{2} \delta_{\sigma \sigma^{\prime}}-\frac{1}{3}\left(c_{i \sigma}^{+} \sigma_{\sigma \sigma^{\prime}} c_{i \sigma^{\prime}}\right)^{2}\right],
$$

may be used (see, e.g. Ref [7]).

It was previously discussed (see, e.g. Refs. [30, 31, 32, 33, 34, 35]), that although the representations (37) and (38) are equivalent, they lead to different effective actions (the so-called Fritz ambiguity). In particular, the representation (37) leads to an action with the scalar auxiliary fields and therefore is not transparently $S U(2)$ invariant. The representation (38) is free from this problem, but does not reproduce correctly the mean-field results for the Hubbard model due to an extra factor of $1 / 3$. The reason for this difficulty is that the factor $1 / 3$ in Eq. (38) takes into account that not only a lowest order component of the longitudinal interaction $\left(c_{i \sigma}^{+} \sigma_{\sigma \sigma^{\prime}}^{z} c_{i \sigma^{\prime}}\right)^{2}$, but also the higher order transverse fluctuations which arise from the $\left(c_{i \sigma}^{+} \sigma_{\sigma \sigma^{\prime}}^{x, y} c_{i \sigma^{\prime}}\right)^{2}$ part of the interaction contribute to the self-energy of the $S^{z}$ field and similar to the other quantities (see, e.g., Ref. [33]).

Below we construct an effective action which is explicitly $S U(2)$ invariant and at the same time reproduces correctly the mean-field results. Although some attempts of derivation of such an action were performed earlier, they were either restricted to systems with additional orbital degeneracy [32] or used additional auxiliary vector field to perform averaging over directions in Eq. (37) 30, 34.

We start from the Hubbard model action 


$$
\begin{gathered}
\mathcal{S}_{\mathrm{H}}=\sum_{k \sigma}\left(-\mathrm{i} \nu_{n}+\varepsilon_{\mathbf{k}}\right) c_{k \sigma}^{+} c_{k \sigma}+\mathcal{S}_{\mathrm{int}}-\sum_{k \sigma}\left(\eta_{k \sigma}^{+} c_{k \sigma}+c_{k \sigma}^{+} \eta_{k \sigma}\right), \\
\mathcal{S}_{\mathrm{int}}=\frac{U}{4 N} \sum_{q}\left(n_{q} n_{-q}-s_{q}^{z} s_{-q}^{z}\right), \mathbf{s}_{q}=\sum_{k \sigma \sigma^{\prime}} c_{k \sigma}^{+} \vec{\sigma}_{\sigma \sigma^{\prime}} c_{k+q \sigma^{\prime}},
\end{gathered}
$$

where we have introduced fermionic source fields $\eta, \eta^{+}$. To derive an effective action we introduce auxiliary field $\mathbf{S}_{q}$ such that

$$
\mathcal{S}_{\text {int }}=\frac{1}{N} \sum_{q}\left\{R_{q}^{-1} \mathbf{S}_{q} \mathbf{S}_{-q}+U_{\mathrm{eff}} \mathbf{S}_{q} \mathbf{S}_{-q}+\frac{U}{4}\left[n_{q} n_{-q}+U_{\mathrm{eff}} \chi_{q}^{0}\left(\mathbf{s}_{q} \mathbf{s}_{-q}\right)-\left(s_{q}^{z} s_{-q}^{z}\right)\right]\right\}
$$

where $R_{q}^{-1}=\left(\chi_{q}^{0}\right)^{-1}-U_{\text {eff }}, U_{\text {eff }}=U /\left(1+U \chi_{q}^{0}\right), \chi_{q}^{0}$ is the bare polarization operator (see Eq. (3) ). It can be shown that the integration over the field $\mathbf{S}_{q}$ leads to the original action (40). Let us now consider the sum of the ladder diagrams, generated by the interaction $U_{\text {eff }} s_{q}^{z} S_{-q}^{z}$. This summation results in the renormalizaton of the $z$-component of the interaction itself $U_{\text {eff }} s_{q}^{z} s_{-q}^{z} \rightarrow U s_{q}^{z} s_{-q}^{z}$, the electron-paramagnon vertex, $U_{\text {eff }} s_{q}^{z} S_{-q}^{z} \rightarrow U s_{q}^{z} S_{-q}^{z}$ and the renormalization of the paramagnon propagator $R_{q} \rightarrow \chi_{q}$, where

$$
\chi_{q}^{-1}=\left(\chi_{q}^{0}\right)^{-1}-U\left(1-U \chi_{q}^{0}\right) .
$$

Introducing the two-particle irreducible interaction $\left(s_{q}^{z} s_{-q}^{z}\right)_{z \text {-irr }}$, where the index $z$-irr means that the ladder diagrams generated by this interaction have to be excluded, the result (41) can be therefore rewritten in the form

$$
\begin{aligned}
\mathcal{S}_{\mathrm{int}}= & \frac{1}{N} \sum_{q}\left\{\chi_{q}^{-1} S_{q}^{z} S_{-q}^{z}+R_{q}^{-1}\left(S_{q}^{x} S_{-q}^{x}+S_{q}^{y} S_{-q}^{y}\right)+U_{\mathrm{eff}}\left(s_{q}^{x} S_{-q}^{x}+s_{q}^{y} S_{-q}^{y}\right) .\right. \\
& \left.\left.+U s_{q}^{z} S_{-q}^{z}+\frac{U}{4}\left[n_{q} n_{-q}-\left(s_{q}^{z} s_{-q}^{z}\right)_{z-\mathrm{irr}}\right]+U_{\mathrm{eff}} \chi_{q}^{0}\left(s_{q}^{x} s_{-q}^{x}+s_{q}^{y} s_{-q}^{y}\right)\right]\right\},
\end{aligned}
$$

Note, that in addition to the spin-fermion interation the action (43) contains the Hubbard interaction irreducible in the $s^{z}$-spin channel. Performing the same steps in $x$ and $y$ spin directions we obtain

$$
\mathcal{S}_{\mathrm{int}}=\frac{1}{N} \sum_{q}\left\{\chi_{q}^{-1} \mathbf{S}_{q} \mathbf{S}_{-q}+U \mathbf{S}_{q} \mathbf{s}_{-q}+\frac{U}{4}\left[n_{q} n_{-q}-\left(\mathbf{s}_{q} \mathbf{s}_{-q}\right)_{\mathrm{ph}-\mathrm{irr}}\right]\right\}
$$

where the subscript ph-irr denotes that the ladder diagrams in longitudinal or transversal particle-hole channel generated by the interaction $\left(\mathbf{s}_{q} \mathbf{s}_{-q}\right)$ are excluded. Consideration of higher order in $U$ contributions to the ladder-type processes leads to the same action (44) with the exact polarization operator $H_{q}$ instead of the bare one $\chi_{q}^{0}$ in the Eq. (42).

The effective model (44) contains both the spin-fermion (first two terms) and the irreducible Hubbard (third term) interaction. Due to its irreducibility the latter is expected to lead only to small renormalization of the parameters of the spin-fermion model and it is neglected in the main text.

To obtain the magnetic susceptibility of the Hubbard model (39) we differentiate the corresponding generating functional (39), (44) with respect to fermionic sources. In this way we obtain two contibutions:

$$
\chi_{q}^{\mathrm{el}}=\chi_{q, \mathrm{SF}}^{\mathrm{el}}+U H_{q}^{2}
$$


where the first term originates from the one-particle reducible diagrams containing the spinfermion interaction $U \mathbf{S}_{q} \mathbf{s}_{-q}$,

$$
\chi_{q, \mathrm{SF}}^{\mathrm{el}}=\frac{H_{q}}{1-U^{2} H_{q} \chi_{q}}
$$

and the second term originates from the irreducible Hubbard interaction. The result (46) is used in main text, Eq. (13).

\section{Appendix B. The Diagram Technique for the Param- agnon Vertex in the Spin-Fermion Model}

To consider spin fluctuations in the spin-fermion model (8) it is convenient to introduce the r-vertex of paramagnon interaction,

$$
\begin{gathered}
\Gamma^{j_{1} \ldots j_{r}}\left(q_{1}, \ldots, q_{r}\right)=-2^{r} T\left(\prod_{i=1}^{r} \chi_{q_{i}}^{-1}\right) \int_{0}^{\beta} d \tau_{1} \ldots d \tau_{r} e^{i \omega_{1} \tau_{1}+\ldots+i \omega_{r} \tau_{r}}\left\langle T_{\tau}\left[S_{\mathbf{q}_{1}}^{j_{1}}\left(\tau_{1}\right) \ldots S_{\mathbf{q}_{r}}^{j_{r}}\left(\tau_{r}\right)\right]\right\rangle_{c} \\
q_{1}+\ldots+q_{r}=0
\end{gathered}
$$

where $q_{1}, \ldots, q_{r}$ are the paramagnon momenta, $j_{1}, \ldots, j_{r}$ are the spin indices. The product $\prod_{i=1}^{r} \chi_{q_{i}}^{-1}$ removes the external Green functions of the field $\mathbf{S}$; for $r>2$ we assume $\chi_{q_{i}} \rightarrow \mathcal{R}_{q_{i}}$ which corresponds to removing the exact propagators of the field $\mathbf{S}$, the average with the index $c$ denotes that the diagrams, for which the external spin operators (47) are connected with each other through the lines corresponding to the electronic Green functions (see below), should be considered. Whereas the 2-vertex describes self-energy corrections to the paramagnon propagator, the higher order vertices describe interaction of the renormalized (physical) paramagnons.

The calculation of correlation functions of spin operators can be carried out using the expansion $\exp \left[-\beta \mathcal{S}_{\text {int }}\right]$ of the functional (8) in the series in electron-paramagnon interaction. According to the Wick theorem, the average in (47) is expressed through all possible combinations of averages of pairs of operators

$$
\left\langle S_{q}^{j} S_{q^{\prime}}^{j^{\prime}}\right\rangle \equiv T \int_{0}^{\beta} d \tau d \tau^{\prime} e^{\mathrm{i} \omega_{n} \tau+\mathrm{i} \omega_{n^{\prime}} \tau^{\prime}}\left\langle T_{\tau}\left[S_{\mathbf{q}}^{j}(\tau) S_{\mathbf{q}^{\prime}}^{j^{\prime}}\left(\tau^{\prime}\right)\right]\right\rangle=\frac{1}{2} N \mathcal{R}_{q} \delta_{q,-q^{\prime}} \delta_{j j^{\prime}}
$$

where $\mathcal{R}_{q}$ is the paramagnon propagator. The corresponding contributions to the vertex can be represented by diagrams, every diagram for the $r$-vertex (47) of the order $n$ consisting of:

- $n$ internal $\left(\sigma^{ \pm}, \sigma^{z}\right), r$ external $\left(S^{ \pm}, S^{z}\right)$ interaction vertices, and $n$ solid lines, corresponding to the electronic Green functions. One electronic line enters and goes out of each internal vertex;

- the longitudinal vertices $\left(\sigma^{z}, S^{z}\right)$ are connected by dashed lines, the transverse vertices $\left(\sigma^{ \pm}, S^{ \pm}\right)$are connected by wavy lines. The external $S^{+}$-vertex is connected with the internal $\sigma^{+}$-vertex, the $S^{-}$-vertex with $\sigma^{-}$, and $S^{z}$-vertex with $\sigma^{z}$; internal wavy lines connect $\sigma^{+}$- and $\sigma^{-}$-vertices, and dashed lines $\sigma^{z}$-vertices;

- The factor $(1 / 2) \mathcal{R}_{q}$ corresponds to each internal dashed or wavy line with the 4momentum $q$, the factor $\mathcal{G}_{k}$ - to each solid line with the 4-momentum $k$; the sum 
of momenta, which enter each internal vertex, is equal to the sum of momenta going out of this vertex, the summation over all independent momenta in the resulting expression being performed;

- The number of internal transverse vertices is even for each loop and $\sigma^{+}$- and $\sigma^{-}$-vertices alternate along the electronic Green function line. The type of all transverse vertices in diagram for which it is possible to determine it unambiguously should be determined;

- The factor corresponding to a diagram is $I^{n}(T / N)^{(n-r) / 2+1}(-1)^{c+f+1} 2^{n_{\perp}+n_{K}}$, where $c$ is the full number of longitudinal vertices, placed between neighboring transverse $\sigma^{-}$- and $\sigma^{+}$-vertices. $f$ is the number of electronic loops, $n_{K}$ is the number of the independent internal transverse vertices, type of which is not determined unambiguously, $n_{\perp}$ is the number of wavy lines;

The lowest-order diagram for the vertex of any order is the electronic loop (10). The vertex renormalization is performed in two ways: the internal paramagnon lines can appear in electronic loop or consist of several loops, connected by paramagnon lines.

To classify diagrams it is convenient to define the one-loop vertex $\Gamma_{1-\text { loop }}^{j_{1} \ldots j_{r}}\left(q_{1}, \ldots, q_{r}\right)$ as a sum of diagrams for the vertex containing only one electronic loop of electronic Green function (see Fig. 2b). Every diagram for the vertex consists of one or more one-loop vertices, connected by paramagnon lines. Comparing coefficients of expansions in the series in $I$ of (47) and (48), it is possible to show that

$$
\begin{gathered}
\Gamma_{1-\text { loop }}^{j_{1} \ldots j_{r}}\left(q_{1}, \ldots, q_{r}\right)=\frac{T I^{r}}{r N} \sum_{\mathcal{P}_{q}} \operatorname{Tr}_{\sigma} \sum_{k_{1} \ldots k_{r}} \\
\left\langle\mathbb{G}_{k_{1}, k_{2}}[\mathbf{S}] \sigma^{j_{1}} \mathbb{G}_{k_{2}+q_{1}, k_{3}+q_{1}}[\mathbf{S}] \sigma^{j_{2}} \ldots \mathbb{G}_{k_{r}+q_{1}+\ldots+q_{r-1}, k_{1}+q_{1}+\ldots+q_{r-1}}[\mathbf{S}] \sigma^{j_{r}}\right\rangle_{S},
\end{gathered}
$$

index $\mathcal{P}_{q}$ points that the sum is taken over all permutations numbers $i$ of momenta $q_{i}$ and simultaneously spin indices $j_{i}$. The explicit expressions for one-loop 2- and 4-vertices are given by formulas (27) and (34) of main text.

\section{Appendix C. The Second Derivative of the Magnetic Susceptibility}

The second derivative of the momentum dependent susceptibility at $T=0$ has a form

$$
\begin{gathered}
\partial_{q_{x}}^{2} H_{\mathbf{q}=0}(\omega=0)=\frac{\sqrt{3 / 8 \pi}}{\Delta^{2}} \times \\
\times \int_{\left(w_{1}-\mu\right) / \Delta}^{\left(w_{2}-\mu\right) / \Delta} d \xi \rho_{p}(\mu+\xi \Delta)\left[\left(-3 \xi^{4}+5 \xi^{2}-8 / 3\right) \exp \left(-3 \xi^{2} / 2\right)-\operatorname{Ei}\left(-3 \xi^{2} / 2\right)\right],
\end{gathered}
$$

where $w_{1,2}= \pm 4 t+8 t^{\prime}, \operatorname{Ei}(x)=\int_{-x}^{+\infty} d t e^{-t} / t, \rho_{p}(\varepsilon)$ is the average value of square of electronic velocity in the iso-energetic surface $\varepsilon_{\mathbf{k}}+\mu=\varepsilon$,

$$
\rho_{p}(\varepsilon)=\frac{1}{N} \sum_{\mathbf{k}} \delta\left(\varepsilon+\mu-\varepsilon_{\mathbf{k}}\right) v_{\mathbf{k}}^{2}=\oint_{\varepsilon_{\mathbf{k}}=\varepsilon+\mu} \frac{d \sigma}{(2 \pi)^{2}} v_{\mathbf{k}}
$$


The calculation of the integral in (50) results in

$$
\begin{gathered}
\rho_{p}(\varepsilon)=\frac{1}{\pi^{2}\left(t^{2}+\varepsilon t^{\prime}-4 t^{2}\right)^{1 / 2}} \times \\
\times\left[\left(-2|\varepsilon| t+4 t^{\prime} \varepsilon-\varepsilon^{2}\right) \mathrm{F}\left(k^{2}\right)+8\left(t^{2}+\varepsilon t^{\prime}-4 t^{\prime 2}\right) \mathrm{E}\left(k^{2}\right)+\frac{\varepsilon^{2} t}{t+2 t^{\prime} \operatorname{sign} \varepsilon} \Pi\left(1-\frac{|\varepsilon|}{4 t+8 t^{\prime} \operatorname{sign} \varepsilon}, k^{2}\right)\right],
\end{gathered}
$$

where

$$
k^{2}=-\frac{\left(-4 t+\varepsilon-8 t^{\prime}\right)\left(4 t+\varepsilon-8 t^{\prime}\right)}{16\left(t^{2}+\varepsilon t^{\prime}-4 t^{\prime}\right)^{2}},
$$

and $\mathrm{F}, \mathrm{E}, \Pi$ are the elliptic integrals first, second and third kind respectively. According to (49) $\partial_{q_{x}}^{2} H_{\mathbf{q}=0}(\omega=0 ; \Delta \rightarrow 0)=-1 / 12 \rho_{p}^{\prime \prime}(\mu)$.

Plots of the functions of $\rho_{p}(\varepsilon)$ and the second derivative of susceptibility with respect to momentum $\partial_{q_{x}}^{2} H_{\mathbf{q}=0}(\omega=0 ; \Delta \rightarrow 0)$ are presented in Fig. 13. The sign of
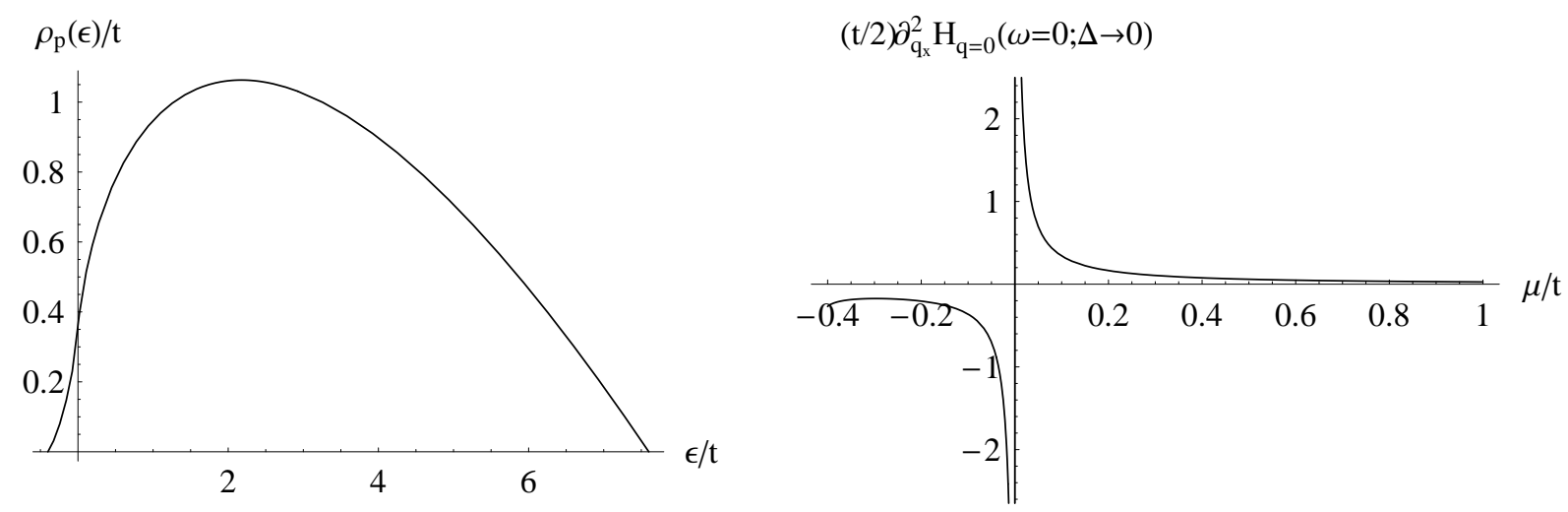

Figure 13: The prot of : (a) $\rho_{p}(\varepsilon) ;\left(\right.$ b) $\partial_{q_{x}}^{2} H_{\mathbf{q}=0}(\omega=0 ; \Delta \rightarrow 0)$ vs $\mu$

$\partial_{q_{x}}^{2} H_{\mathbf{q}=0}(\omega=0 ; \Delta \rightarrow 0)$ coincides with the sign of $\mu$. Hence, the susceptibility of noninteracting electrons has its local maximum in the point $\mathbf{q}=0$ for electronic concentration $n<n_{\mathrm{VH}}$ and minimum for $n>n_{\mathrm{VH}}$. The second derivative of the susceptibility with respect to momentum tends to plus (minus) infinity as the electronic concentration approaches the Van Hove filling from the above (below). 


\section{References}

[1] J. Bednorz and K. F. Muller, Z. Phys. B 64, 189 (1986).

[2] I. I. Mazin and D. J. Singh, Phys. Rev. Lett. 79, 773 (1997).

[3] N. Kikugawa and Y. Maeno, Phys. Rev. B 70, 134520 (2004).

[4] F. Nakamura, T. Goko, M. Ito et al., Phys. Rev. B 65, 220402 (2002).

[5] K. K. Murata and S. Doniach, Phys. Rev. Lett. 29, 285 (1972).

[6] I. E. Dzyaloshinskii and P. S. Kondratenko, Sov. Phys. JETP 43, 1036 (1976).

[7] T. Moriya, J. Phys. Soc. Jpn. 40, 933 (1976); T. Moriya, Spin Fluctuations in Itinerant Electron Magnetism, Springer: Berlin, 1985.

[8] J. A. Hertz, Phys. Rev. B 14, 1165 (1976).

[9] A. J. Millis, Phys. Rev. B 48, 7183 (1993).

[10] D. Belitz, T. Kirpetrick, and T. Vojta, Phys. Rev. B 55, 9452 (1997); A. V. Chubukov, C. Pepin, and J. Rech, Phys. Rev. Lett. 92, 147003 (2004); A. V. Chubukov, C. Pepin, and J. Rech, Phys. Rev. B 74, 195126 (2006).

[11] See, for example, the results of F. Onufrieva, P. Pfeuty, and M. Kiselev, Phys. Rev. Lett. 82, 2370 (1999); F. Onufrieva and P. Pfeuty, Phys. Rev. B 61, 799 (2000)

[12] J. A. Hertz and M. A. Klenin, Phys. Rev. B 10, 1084 (1974).

[13] P. Monthoux and D. Pines, Phys. Rev. B 47, 6069 (1993).

[14] J. Schmallian, D. Pines, B. Stoikovic, Phys. Rev. B. 60, 667 (1999).

[15] E. Z. Kuchinskii and M. V. Sadovskii, Zh. Eksp. Theor. Phys. 115, 1765 (1999) [Sov. Phys. JETP 88, 968 (1999)]; M. V. Sadovskii, Usp. Fiz. Nauk. 171, 539 (2001) [Physics Uspekhi 44, 515 (2001)]

[16] A. A. Katanin, Phys. Rev. B 72, 035111 (2005).

[17] A. A. Katanin and A. P. Kampf. Phys. Rev. B 68, 195101 (2003).

[18] H. Tasaki, Phys. Rev. Lett. 69, 1608 (1992); A. Mielke and H. Tasaki, Commun. Math. Phys. 158, 341 (1993).

[19] S. V. Vonsovskii, M. I. Katsnelson, and A. V. Trefilov, Phys. Met. Metallogr. 76, 247 (1993).

[20] A. Auerbach, Interacting Electrons and Quantum Magnetism, Springer-Verlag: N.Y, 1994.

[21] N. D. Mermin, H. Wagner, Phys. Rev. Lett. 17, 1133 (1966); T. Koma, H. Tasaki, Phys. Rev. Lett. 68, 3248 (1992).

[22] F. Guinea, Nucl. Phys. B 642, 407 (2002). 
[23] J. Vilk and A.-M. S. Tremblay, J. Phys. I (France) 7, 1309 (1997).

[24] A. A. Katanin, A. P. Kampf, and V. Yu. Irkhin, Phys. Rev. B 71, 085105 (2005).

[25] S. Ma, Modern Theory of Critical Phenomena, W. A. Benjamin: London, 1976.

[26] A. A. Katanin and V. Yu. Irkhin, E-print archives, cond-mat/0602657.

[27] S. L. Adler, Phys. Rev. B, 137, 1022 (1965).

[28] V. G. Baryakhtar, V. N. Krivoruchko and D. A. Jabonsky, Green Functions in the Theory of Magnetism [in Russian], Naukova Dumka: Kiev, 1984.

[29] J. Kanamori, Progr. Theor. Phys. 30, 275 (1963).

[30] H. J. Schulz, Phys. Rev. Lett. 65, 2462 (1990).

[31] Z. Y. Weng, C. S. Ting, and T. K. Lee, Phys. Rev. B 43, 3790 (1991).

[32] P. K. Maiti and A. Singh, Phys. Rev. B 49, 6078 (1994).

[33] N. E. Bickers and D. J. Scalapino, cond-mat/0010480 (unpublished).

[34] N. Dupuis, Phys. Rev. B 65, 245118 (2002); K. Borejsza and N. Dupuis, Phys. Rev. B 69, 085119 (2004).

[35] T. Baier, E. Bick, and C. Wetterich, Phys. Rev. B 70, 125111 (2004). 